Marquette University

e-Publications@Marquette

College of Nursing Faculty Research and

Publications

Nursing, College of

$1-1-2002$

Resolving the Uncertainty of Preterm Symptoms: Women's Experiences With the Onset of Preterm Labor

Marianne E. Weiss

Marquette University, marianne.weiss@marquette.edu

Nancy P. Saks

National University

Susan Harris

National University

Accepted version. Journal of Obstetric, Gynecologic, \& Neonatal Nursing, Vol. 31, No. 1 (January 2002): 66-76. DOI. (C 2002 Blackwell Publishing. Used with permission. 


\title{
Resolving the Uncertainty of Preterm Symptoms: Women's Experiences with the Onset of Preterm Labor
}

\author{
Authors: Marianne E. Weiss, RN, DNSc, Nancy P. Saks, RN, DNSc, Susan Harris, RN, \\ DNSc
}

\begin{abstract}
:
Objective: To describe expectant women's experiences with the onset of preterm labor. Design: Qualitative, using grounded theory methods.

Setting: Southwestern tertiary women's hospital.

Participants: Thirty pregnant women who were less than 35 weeks gestation, had experienced preterm labor within the past 7 days, and had no previous experience with preterm labor.

Data Source: Taped and transcribed interviews.

Results: Themes that emerged from the interview data included the following: recognition and naming of sensations, a consistent pattern of attribution of symptoms, the threat or risk inferred by the attributed cause of the symptom pattern, the associated certainty or uncertainty about these attributions, the process of interpreting and verifying symptom meaning, and the decision to self-manage the symptoms or engage health care assistance. The core process of women experiencing the onset of preterm labor symptoms was identified as "resolving the uncertainty of preterm labor symptoms: recognizing and responding to the possibilities."
\end{abstract}

Conclusions: Preterm labor often is not within expectant women's consciousness. They may attribute the symptoms to nonthreatening causes, which results in delays in seeking care for preterm labor. Education about symptom patterns at the onset of preterm labor will increase the probability that women and their health care providers will recognize and interpret the early, subtle symptoms that herald the onset of preterm labor. Uncertainty in illness theory and attribution theory offer frameworks for understanding women's experiences with the onset of preterm labor.

Despite advances in risk assessment, diagnostic strategies, and prevention-focused care, the rate of preterm birth has increased over the past three decades to $11.6 \%$ in 1998 (Ventura, Martin, Curtin, Mathews, \& Park, 2000). Early detection and intervention are the cornerstones of preterm birth prevention efforts. The recognition of preterm labor at an early stage requires that the expectant woman and her health care provider identify the subtle symptoms of preterm labor. Preterm birth prevention educational programs have included education about the warning signs of preterm labor but reach only a small portion of the pregnant population.

1 Weiss, Saks, \& Harris 
Information in consumer literature and childbirth preparation classes often does not differentiate between preterm labor and the symptom patterns of term labor. Furthermore, the focus of women's advocacy groups on the normalcy of pregnancy and childbirth has decreased awareness and diverted attention from the possibility of adverse occurrences such as preterm labor. Preterm birth prevention programs for women at high risk have used self-monitoring of symptom patterns, weekly cervical examinations, telephonic monitoring, home visiting, and home uterine activity monitoring, alone or in combination, with mixed results (Collaborative Group on Preterm Birth Prevention, 1993; Dyson et al., 1998; Holbrook et al., 1987; lams, Johnson, \& Hamer, 1990; lams, Johnson, \& O’Shaughnessy, 1988; Nagey, Bailey-Jones, \& Herman, 1993; U.S. Preventive Health Services Task Force, 1993).

Subtle changes in uterine activity patterns have been detected a few days to several weeks before the overt onset of preterm labor (Cooper et al., 1990; Katz, Newman, \& Gill, 1986). The similarity of preterm labor symptoms to normal or expected pregnancy discomforts complicates the recognition of preterm labor (Freston et al., 1997). Delays in accessing health care may result from confusion about the meaning of subtle and nonspecific symptoms. The purpose of the current study was to explore women's experiences with the onset of preterm labor-related symptoms. Through this exploration, the goal was to capture the subtleties of symptom experiences associated with the prodrome of preterm labor and develop an understanding of the processes that lead to recognition and decisions about seeking health care intervention. Specific research questions were the following:

1. How do expectant women describe the experience of the onset of preterm labor?

2. How do expectant women describe and interpret the initial cues of preterm labor?

3. What resources do expectant women use in interpreting and making decisions about preterm labor cues?

4. What processes occur in making an appraisal of preterm labor cues and a decision to seek health care?

\section{Literature Review}

Medical research about preterm labor recognition has focused on the frequency of symptoms and detection of increased uterine activity. The frequency and type of symptoms have been reported in several studies (Cooper et al., 1990; lams, Johnson, \& Parker, 1994; lams, Stilson, Johnson, Williams, \& Rice, 1990; Katz, Goodyear, \& Creasy, 1990; Kragt \& Keirse, 1990). Women who develop preterm labor have significantly more symptoms preceding preterm labor

2 Weiss, Saks, \& Harris 
than women of the same gestational age who do not develop preterm labor (lams, Stilson, et al., 1990; Katz et al., 1990). Commonly reported symptoms included painful contractions, painless contractions, menstrual cramps, backache, pelvic pressure, diarrhea, and increased vaginal discharge and frequency of urination (Maloni, 2000). Although symptoms are evident in the week before the onset of preterm labor, an increase in uterine activity heralding preterm labor occurs within 24 hours prior to labor onset (lams et al., 1994; Katz et al., 1986). Most of the symptoms, when evaluated prospectively, were not predictive of subsequent preterm labor, which suggests that routine assessment of preterm labor-related symptoms may not be useful in the early identification of women who would develop preterm labor (Cooper et al., 1990).

The pregnant woman's early detection of preterm labor is key to early diagnosis and treatment, but her identification of its subtle symptoms is often incomplete and inaccurate. Newman, Gill, Wittreich, and Katz (1986) reported that expectant women perceived on the average only $15 \%$ of their preterm contractions. In a study of high-risk patients on home uterine activity monitoring diagnosed with preterm labor, $24 \%$ had been identified by symptoms only, $24 \%$ by increased uterine activity (per monitor) in the absence of symptoms, $43 \%$ by a combination of uterine activity and symptom pattern, and $10 \%$ by cervical examination during a routine office visit. Two thirds of the women were able to identify symptoms associated with the onset of preterm labor (lams, Johnson, et al., 1990). Kragt and Keirse (1990) found that $75 \%$ of patients seeking care for evaluation of preterm labor signs and symptoms correctly interpreted the threat associated with their symptoms and progressed to give birth or to be admitted to the hospital for more than 24 hours for the management of preterm labor.

Collectively, these studies suggest that expectant women experience a range of symptoms during the prodromal period before the onset of preterm labor. Although these symptoms may not be highly predictive of preterm labor onset, expectant women do make interpretations of their symptom experience that lead them in most cases to seek health care assistance appropriately. Not addressed in these studies are the way in which women with preterm labor symptoms interpret these symptom patterns when they are actually experiencing them and the factors that support or interfere with recognition of preterm labor and health care seeking. None of these studies addressed the relationship between the nature of the symptom experience and decisions to seek health care services for the management of preterm symptoms.

Three qualitative studies about recognizing and seeking care for preterm labor have been conducted. Findings of these studies include expectant women's confusion with early symptoms, self-management of symptoms, and lay consultation prior to seeking health care for preterm labor.

3 Weiss, Saks, \& Harris 
Mackey and Coster-Schulz (1992) described two paths to care seeking for preterm labor symptoms. "Waiters" were women who sought care after waiting a period of 2 days to 2 weeks. These women typically sought care when self-care measures became ineffective. "Nonwaiters" were women who sought care within a few hours of initiation of symptoms, either calling their health care provider or going directly to the hospital. Both waiters and nonwaiters used lay consultants for advice on appropriate actions.

A pattern of self-diagnostic confusion with preterm labor symptoms was identified in a qualitative study of women experiencing preterm labor (Patterson, Douglas, Patterson, \& Bradle, 1992). Ambiguous symptoms, absence of meaningful labels to attach to symptoms, and the context of the pregnancy with its expected discomforts created a situation of confusion in understanding preterm labor symptoms. Women attempted to make sense of their symptoms by comparing them with the personal expectations and experiences of others, gathering data, and seeking information. Professional care seeking was used as a strategy of last resort when symptoms could no longer be self-managed.

The beginning of the preterm labor experience has been described as "feeling unbalanced." Women described a personal sense of knowing that all was not well as they began to experience a variety of physical feelings or symptoms. These feelings were interpreted by some women as worrisome and by others as just another of the many symptoms experienced during their pregnancy. Care seeking occurred when the symptom pattern increased or changed (Coster-Schulz \& Mackey, 1998).

\section{Theoretical Framework}

In planning the study, the researchers, who had substantial experience in working with expectant women, explored their beliefs about symptom experiences and preterm labor recognition. Although qualitative research is often characterized by the absence of a theoretical framework, Thorne, Kirkham, and MacDonald-Emes (1997) suggested that a framework based

on a critical analysis of the existing body of knowledge represents an appropriate foundation on which to build qualitative research designs. Serving as a starting point for the inquiry rather than a structure for analysis, a theoretical framework offers a platform for expanding on prior knowledge, challenging prior conceptualizations, and creating new interpretations of the meaning of human health experiences.

Explication of the researchers' assumptions resulted in a recognition that Mishel's (1988) "theory of uncertainty in illness" provided a framework of concepts consistent with clinical

4 Weiss, Saks, \& Harris 
expressions of the experience of expectant women in recognizing and seeking care for preterm labor symptoms. Mishel's uncertainty in illness theory is a model for understanding the way in which individuals create meaning of illness-related stimuli. Within the limits of his or her cognitive capacity, the individual processes the elements of the stimuli frame (including symptom pattern, event familiarity, and event congruence) and input from available structure providers (social support, credible authority, and education) to construct an appraisal or interpretation of the illness event. The individual experiences uncertainty if unable to form a cognitive schema. Appraisal of the uncertain events, through inference or illusion, evokes coping strategies to resolve the uncertainty, leading to effective or ineffective adaptation.

\section{Research Design}

Because the purpose of the study was to explore and describe women's experiences with the onset of preterm labor, a qualitative design using a grounded theory approach was selected. The concepts of Mishel's theory provided a starting point for exploration of women's experiences with the onset of preterm labor symptoms. Interview questions were developed to open dialogue concerning the experiences and meanings of symptoms and events leading to admission for treatment of preterm labor. Interviews began with a request of the participant to describe the days before she came to the hospital. The researchers made a conscious effort during interviewing to follow women's stories about their experiences and take new directions as content emerged from these stories. Explorations of experiences were pursued beyond the confines of the concepts of uncertainty theory as thematic trajectories emerged from the participants' stories. Probing for depth, clarity, and extension encouraged emergence of elements of the experience beyond, different from, or contrary to those suggested within the theoretical framework.

The sample for the study consisted of 30 women who were admitted to the hospital with a diagnosis of preterm labor. Participants had no previous experience with preterm labor in current or prior pregnancies, were less than 35 weeks gestation, had been admitted within the past 7 days, and spoke English. Twenty-eight (93\%) of the participants were admitted for suppression of preterm labor symptoms, and the contraction pattern had stabilized before the interview.

Twenty-six (87\%) of these women received tocolytic therapy, and 2 women were managed with bed rest. Tocolytic therapy was not successful with 2 women, who delivered before the interview. Ten (33\%) participants were 20 to 30 weeks gestation at the time of admission. The other 20 (67\%) participants were 30 to 34 weeks gestation. Eight participants (27\%) were dilated $2 \mathrm{~cm}$ or more; 9 (30\%) showed signs of effacement or dilation less than $2 \mathrm{~cm} ; 8$ (27\%) had a closed cervix; 
and $5(17 \%)$ had bleeding that accompanied the preterm labor symptoms, which led to deferral of their examination at admission. Twenty-seven (90\%) participants reported that they experienced uterine contractions before their hospital admission. Three of the 5 participants who had ruptured membranes did not feel contractions before arrival at the hospital and were surprised when told they were having contractions. Eight (27\%) participants experienced both contractions and bleeding.

The sample was diverse in several demographic characteristics. The mean age of the participants was 27.5 years, with a range of 19 to 39 years. Seventeen participants (57\%) were employed outside the home. Twenty-one (70\%) participants were white, 5 (17\%) were Latina, 1 (3\%) was African American, and 3 (10\%) were Asian. Nine (30\%) participants were college graduates, 8 (27\%) had completed junior college, 8 (27\%) were high school graduates, and 2 $(7 \%)$ had attended high school but not graduated. The educational status was unknown for 3 (10\%) participants. Seventeen of the 30 (57\%) women were married, and 17 (57\%) were primiparae.

The study was conducted in a large tertiary-level hospital in a southwestern metropolitan area. Approval to conduct the study was obtained from the hospital's institutional review board. Participants were enrolled in the study after providing informed consent. The interviews took place in each participant's room after the preterm labor symptoms stabilized and were tape recorded with the woman's knowledge. Most of the interviews were completed within 72 hours after admission. The transcript of each interview was verified by one of the researchers. Demographic data were collected from the medical record.

A constant comparative method of analysis was employed. The researchers met regularly to review data and conduct ongoing analysis as interviews were completed. Data collection progressed until the researchers felt they reached saturation of the data. Emerging themes were discussed, and the interview guide was revised to include probes for verification of themes and of content areas emerging during the interviews. Content analysis was accomplished without an a priori coding schema. Two of the researchers coded each of the first three transcripts to develop a coding scheme. Subsequent transcripts were coded independently by one of the two researchers. The researchers discussed coding congruences and divergences on an ongoing basis to develop a consensus on the coding scheme and thematic structure. During the process of coding, previously coded transcripts were reviewed and the coding revised as new or clarified themes emerged. The Ethnograph computer software (Qualis Research Associates, Salt Lake City, UT) was used to assist with data sorting and management. The third investigator served as a

6 Weiss, Saks, \& Harris 
consultant, reviewing the data for validation of interpretation.

\section{Results}

The most striking themes to emerge included the following: recognition and naming of sensations, a consistent pattern of attribution of symptoms, the threat or risk inferred by the attributed cause of the symptom pattern, the associated certainty or uncertainty about these attributions, the process of interpreting and verifying symptom meaning, and the decision to self-manage the symptoms or engage health care assistance. As analysis proceeded, it became clear that the overriding theme or core process in women's experiences with the onset of preterm labor was "resolving the uncertainty of preterm labor symptoms: recognizing and responding to the possibilities." The process involved a dynamic interaction of recognition and attribution of symptoms, certainty or uncertainty about symptom attribution, and the level of perceived threat that shaped expectant women's decisions about symptom management through self-monitoring and self-management or seeking health care assistance.

\section{Recognizing and Naming Symptoms}

The early symptoms of preterm labor are subtle, intermittent, and not easily recognizable as preterm labor. Women labeled these symptoms as pains, cramps, stomachache, pressure, tightness, bloating, soreness, stretching, burning, pushing, and difficulty walking. Pains were often described as sharp, with a feeling of ripping or pulling apart in the lower abdominal area, and as continuous, not rhythmical. One participant commented, "It never got to the point of being rhythmical. It was one continuous feeling." Cramping was described as menstrual-like or cramping in the groin and legs. Primigravidae rarely called these sensations contractions. Having experience with previous contractions did not necessarily mean that symptoms of preterm labor were recognized or labeled as contractions. Multigravidae reported that these sensations were not similar to their recollections of term labor.

Several women described other symptoms occurring in conjunction with the onset of labor. Back pain was described as sharp, knife-like, stabbing, sore, achy, dull, or pressure. Women described diffuse symptoms, such as not being able to find a comfortable position, having difficulty sleeping, being tired, feeling flu-like, or just not feeling right. As one patient stated, "I didn't feel quite right. Something was off balance but I couldn't put my finger on it." Women also described sensations of wetness, dribbling, leaking, or being unable to control urine flow. These sensations were not always described as associated with rupture of membranes or labor. Bleeding was more clearly recognized and referred to specifically as bleeding or spotting.

7 Weiss, Saks, \& Harris 


\section{Creating Meaning of Symptoms: Attribution of Symptoms}

Women described having symptoms related to preterm labor as much as 2 weeks before hospital admission for treatment of acute preterm labor. Many women, however, also described similar symptoms occurring at earlier points in the pregnancy. By attributing symptoms to events or situations, both related and unrelated to the pregnancy, the women created the meaning of symptoms. Women experiencing the early subtle sensations of prodromal preterm labor associated the symptom pattern with a known or expected symptom event or pattern. For example, sensations felt in the abdomen were frequently attributed to Braxton-Hicks contractions, a symptom pattern expected during pregnancy that is reinforced as normal for pregnancy by prenatal classes and consumer literature. Many of the symptoms experienced during the prodrome of preterm labor were attributed to familiar sensations: "I hadn't had a bowel movement in a long period of time and I thought my tummy was plugged up." "I thought maybe the baby was kicking or moving." Some women recognized the increase in contraction activity but attributed the onset to an increase in their own physical activity in the period immediately preceding the arrival of symptoms. "Just doing too much. I had done maternity stretching exercises the night before and I thought maybe I just pulled a muscle."

Rupture of membranes often was interpreted as urinary incontinence. A woman who had experienced leaking for about 10 days noted the following:

I just felt that I had some incontinence because I had already experienced the sneezing and loss of bladder control that I had with my first child. I would feel a little trickle come out and down my leg and just thought that I had just gotten kicked and I just couldn't hold it.

These women noticed the symptoms but attributed them to nonthreatening events or situations. In their minds, they felt they understood the cause of the sensations they were experiencing and only questioned the attribution if symptoms continued or information from other sources invalidated their constructed meaning.

Other symptoms, such as nausea, vomiting, feeling hot, and not feeling well or right, often were attributed to an illness not related to pregnancy: "Just like the flu." or "I thought it was something I ate." Such symptoms were considered either a minor problem that was nonthreatening to the pregnancy or as a problem with significant potential threat to the pregnancy. If the symptom pattern included sharp pains or bleeding, symptoms were usually immediately interpreted as problematic and potentially threatening to the mother or baby, or both.

Only rarely were symptoms attributed to preterm labor. Many women described

8 Weiss, Saks, \& Harris 
characteristic symptoms of preterm labor that had developed progressively over hours or days. Concern for the well-being of self and baby surfaced during this time. However, these women were often surprised when hospital personnel indicated that they were experiencing preterm labor contractions. Preterm labor is a term used by health care providers but not by pregnant women. When women recognized what health care providers would call preterm labor, they referred to it as "losing the baby" or "having the baby early." They used the term "contractions" but rarely "labor contractions," suggesting that women view labor as an event associated with the end of the normal pregnancy period.

The types of attributions noted above refer to women's perceptions of the immediate causes of preterm symptoms. At a deeper level, women questioned why this was happening to them. Several women identified stress associated with the pregnancy as a possible factor underlying the onset of preterm labor.

I have 2 daughters and l'm going through custody for them, divorce with my ex-husband, and trying to get remarried and having a baby with him too. Not knowing how my daughters will react. The money situation. I think about it [stress] a lot too. My daughters weren't stressful pregnancies. Am I having this one early because I was so stressed out?

Some women also felt that having the baby early was a punishment from God or something they had done wrong.

I smoked in my pregnancy this time. So I thought, oh God was punishing me because I smoked. That's why I thought maybe it was underdeveloped.

\section{Certainty/Uncertainty of Symptom Attributions}

The process of creating meaning involved either immediately recognizing the symptom pattern and its associated meaning or searching for meaning. This searching process included ruling in or out the many possible explanations for the symptom experience, searching for possible options, and comparing the current symptoms with knowledge gained through previous experience, experiences of others, reading, or educational classes or materials. Attempts to resolve the uncertainty consisted of searching for additional cues, adding clues together, and synthesizing knowledge from personal experiences and the experiences of others. Emerging symptom patterns, characterized by progressive or repetitive symptoms, supported the continued appraisal and reappraisal of symptoms and modification of the created meaning.

I had a little bit of cramping in the abdominal area, and so I went to the 9 Weiss, Saks, \& Harris 
bathroom and I had a little bit of diarrhea and then I felt ill. I thought it was the stomach flu. The next day, I felt fine, and we went for a drive. When the backache came, I thought it could just be from sitting in the car too much because your back naturally hurts in some seats. And then when my water kept coming out, then I got a clue. That put it all together.

Uncertainty emerged as a complex mix of hope, worry, denial, or failure to recognize the possibility of labor.

Walking around-they would relieve some if I walked around. So I'm like, well maybe they're not. And I'm having a battle with myself trying to decide if they were or they weren't. Because I could make them go away by rest. So I figured, I can't be in labor. So I didn't know. But they kept on coming back and I figured maybe I better get it checked.

\section{Perceived Threat}

Defining the perceived cause of the symptoms was accompanied by an appraisal of the risk or threat to self or fetus.

For about 24 hours I had been having pain, basically felt like heavy menstrual cramps. The reason I didn't think anything of it was because it was continuous. It wasn't contractions, then go away, then contractions. It was just continuous-achy back, lower back, and around my middle belly. It was very, very tight. It didn't really soften up and be squishy like most of the time. So I let it go on for a whole day, from one whole day until the next morning before I finally decided, and the reason I wasn't alarmed was because I felt the baby moving. It was kicking, so I felt it's fine. I was thinking I had gas or I was constipated or just overtired and needed to rest.

Even when symptoms were perceived as potential threats, women often waited to seek medical intervention until the threat was considered real and immediate. A critical point or event often was identified as signaling the reality of the threat. A patient with a history of spotting and cramping for 4 weeks, for which she had been evaluated by her physician and reassured that it was not problematic, described her thoughts during 3 days of heavier bleeding and cramping before contacting her physician again:

I thought I was going to lose it. I wasn't quite sure because I didn't know if the baby was deformed. And then I thought, maybe it was just my cervix acting up or that maybe everything is just in my head or that the umbilical cord somehow got loosened, or I had a million things going through my mind but I was determined nothing bad should happen till my water broke.

Preterm labor was frequently not within the range of possibilities when women considered

10 Weiss, Saks, \& Harris 
options for the cause of their symptoms. Many women said that, as they reflected on the pregnancy experience, they never considered that the pregnancy might end early: "I just never made the connection because it was so early. I just never really knew the connection." "I've been so healthy that having the baby early was not something that I even figured was in the realm of things that were going to happen."

\section{Interpreting/Verifying Symptom Meaning}

Women used a variety of solicited and unsolicited sources of information and support in the process of creating meaning and understanding the significance of the symptom pattern. When symptom patterns emerged, these expectant women confided in their husbands, mothers, sisters, friends, and coworkers about their symptoms, interpretations, and fears. "I did ask my sister. She had two girls and she said that with her first she felt cramping. But she said she thought she was in labor 20 times before she was. So it didn't concern me a lot." If the symptom meaning was certain, the woman's story might be relayed to others in passing and without soliciting information or validation. If the symptom pattern was uncertain or perceived as a threat, the women selected one or more confidantes and consultants from whom they sought information and opinions as well as clarification of personal interpretations of the symptoms. The lay consultation patterns of white and Latina women differed. White women were likely to consult husbands or coworkers for advice, whereas Latina women were more likely to consult their mothers or other female relatives. Even when no solicitation of assistance occurred, these supportive individuals would sometimes provide their own interpretation of the symptom pattern and causative attributions. In these cases, the certainty of interpretation might be replaced by the confusion of uncertainty. White women were more self-directed in deciding to seek medical consultation, whereas Latina women often sought medical care at the urging of their families.

Educational resources provided information to assist women in structuring an interpretation of their symptom experience. Women's knowledge about pregnancy was enhanced by classes, education provided by their health care professional, and reading materials about normal pregnancy and the unexpected complications of pregnancy. This knowledge was used as a benchmark against which to compare symptoms experienced during the pregnancy. However, preterm labor often was not recognized as a possibility and not considered in the woman's differential analysis of her symptoms.

I just assumed that everything was OK because the book said to anticipate lower back pain. It said when you're having contractions it starts in the back and moves to the front and around the side. Mine started in the small of my back and come right up my hipbone. I just thought it was something that

11 Weiss, Saks, \& Harris 
everybody gets in their 3rd trimester.

Delays in recognizing preterm labor symptoms occurred as women attributed them to other causes, until symptoms had progressed to the point at which the original interpretation was no longer congruent with the subsequent symptom pattern.

\section{Managing Symptoms}

The pattern of response to the symptom experience depended on the attribution of the symptoms and the related perceived threat to self or the fetus. In cases in which the symptoms were attributed to a potentially threatening source, the woman usually initiated contact with a health care provider to resolve uncertainty about the symptom pattern or validate the perceived threat. Precautionary health care seeking was used by those who were uncertain about symptom meaning and therefore were not able to assess threat. Even with low degrees of uncertainty, some women wanted their attributions and inferences about the symptom pattern validated.

If a threat was not perceived, women began self-monitoring and self-care measures to validate the certainty of the interpretation and reduce the level of symptoms. For example, many women became increasingly vigilant, monitoring existing symptoms and any other developing sensations. The heightened vigilance resulted in their reevaluation of symptom interpretations as the symptom patterns developed over time and became evident. An increase or decrease in the frequency and intensity of the symptoms led to either continuing certainty or developing uncertainty about the initial symptom meaning. With the onset of new symptoms, women needed to reassess the meaning and perceived threat.

The women began self-care measures when they perceived symptoms as nonthreatening and attributed them to normal pregnancy, increased activity, or nonpregnancy related illness. For example, expectant women attempted to manage their symptoms by increasing rest periods, putting their feet up, rubbing their back or abdomen, taking a bath, using a heating pad, reducing planned activities, increasing fluids, improving nutrition, or self-medicating to reduce pain or manage stomach upset. Only when the symptoms were unresponsive to these self-management strategies would the women seek health care assistance.

\section{Engaging Health Care Assistance}

Women engaged health care assistance for clarification of symptom experiences or validation of their interpretations of symptom meaning. In highly certain situations with no perceived threat, interaction with a health care provider was not sought. In situations of uncertainty, precautionary health care seeking was initiated to provide verification or clarification of symptom meanings. In situations characterized by certainty and perceived threat, the provider 12 Weiss, Saks, \& Harris 
was contacted with very little delay. If the provider was not responsive or created uncertainty by minimizing the perceived threat, the woman continued to seek resolution of the uncertainty and threat through other avenues of health care assistance, such as the hospital labor unit or emergency room.

In seeking clarification and validation of the meaning of the symptom experience, women frequently described to their health care provider not only the symptom pattern but also to what they attributed the symptoms. The providers, in return, frequently validated both the symptom experience and the attribution as common and normal variants of pregnancy, thus minimizing the woman's interpretation of the potential importance of the symptoms. Her uncertainty resolved and the normalcy of the symptoms validated, the woman then interpreted future symptoms as similarly nonthreatening until the symptom pattern developed to a point at which it differed substantially from the earlier pattern.

I thought they were gas pains. I didn't know how to describe them at all to my doctor. I called him and he asked me what was wrong. I thought I was having gas pains. I just hurt all over. I thought, possibly the muscles. I tried stretching exercises and everything and that didn't work. He told me to take something to relieve the pain and not to worry. He told me to have bed rest and come in in the morning if it continued.

Admission to the hospital for preterm labor symptoms occurred when the woman and her provider both agreed that the symptom pattern might be attributed to the onset of preterm labor. Women who had substantial concern about the threat to their pregnancies self-referred to hospitals or urgent care facilities. In these cases, if a physical examination did not validate the onset of preterm labor, the woman received validation of the normalcy and nonthreatening nature of her symptoms. This validation of the symptoms as nonproblematic resulted in delays in care seeking for future symptom patterns. A patient who had been leaking for 10 days reported the following:

I had little bits of pink all over my pad. When I came to the hospital, it had stopped. She monitored the babies, and I was having some small contractions. Then, she did a sample with the litmus paper, and I was half way in between. So she couldn't be definitive about if it was fluid or urine. Then they tried to do a slide culture and couldn't get enough liquid to do it. So they called the doctor and sent me home. The next morning I felt a little cramping in my lower abdomen. ... Then just horrible cramps. I told my husband that they [the physician and nurse] had said it looks fine, but I'm not sure everything's right. Just not convinced.

13 Weiss, Saks, \& Harris 


\section{Discussion}

Women's experiences with the onset of preterm labor reflected the key concepts of Mishel's (1988) uncertainty in illness theory (see Table 1). There is a marked lack of familiarity with the symptom pattern of preterm labor. Even previous term labor does not provide a good experiential background for recognizing preterm labor. Women described the many sensations associated with preterm labor but often failed to associate them with contractions or labor. Women often used known frames of reference and attributed their symptoms to the familiar or expected sensations associated with normal pregnancy, excessive activity, or an illness unrelated to the pregnancy. Some women were certain of their attributions until the symptom pattern progressed. Others continued to search for resolution of their uncertainty. Few women were aware of the possibility of preterm labor, and those who knew about preterm labor did not believe the possibility was relevant for their situation. Women coped with the uncertainty and perceived threat of preterm symptoms by asking others for interpretation or verification of their symptoms, initiating self-care measures to manage the symptoms, and engaging health care assistance. Adaptation, the last stage of the uncertainty in illness theory, occurred when the woman was admitted for treatment of preterm labor, which resulted either in delay of the preterm delivery or in a preterm birth.

As themes emerged from the data, it became apparent that attribution theory also could provide a useful framework for understanding women's experiences with the onset of preterm labor. Attribution is the process by which inferences are made about the causes of behavior. It is a process by which a common person, as opposed to a professional, searches for an understanding of the causes of behavior (Shaver, 1983). In relation to health and illness, attribution is a lay explanation of illness. Nearly all patients develop some attributions about the cause of their condition (Watts, 1982).

Kelley (1971) identified three types of information used in making attributions: consistency information (similarity to past circumstances), consensus information (similarity to experiences of others), and distinctiveness information (specific to a particular illness). These informational inputs are similar to Mishel's elements of the stimuli frame (including event familiarity, event congruence, and symptom pattern). Weiner (1979) indicated that perceived causes of illness can be categorized by their stability (long lasting or subject to change), location (internal or externally caused), and control (ability of the individual to control the cause). Together, these three factors lead to attributions to the following: (a) an internal/stable/uncontrollable cause, such as a problem pregnancy or heredity; (b) an internal, unstable, controllable cause, such as health habits in

14 Weiss, Saks, \& Harris 
pregnancy or excessive activity; (c) an external/stable/uncontrollable cause or life stress events, such as job, family illness, or marital problems; and (d) an external, unstable, uncontrollable cause, such as viral infection. These attributions lead to individual perceptions of vulnerability and illness severity and the perceived threat of preterm labor and neonatal death. Modifying and enabling factors such as protective coping strategies (self-care behaviors, rest, and increased vigilance) and input from other credible sources influence decisions to self-manage or seek professional health care assistance (Furnham, 1988; Jaspers, 1983; Pendleton, 1983).

Mishel's theory of uncertainty in illness and attribution theory enhance the understanding of the women's experiences in recognizing and seeking care for preterm labor symptoms. Although Mishel's model includes the concepts of inference, the specific nature of the causal attribution from stimuli frame inputs is important in creating the individual interpretation of the perceived threat that leads to care-seeking actions. Likewise, the notion of uncertainty adds to the explanatory power of attribution theory. For example, some women clearly attribute their symptoms to a definitive cause. Certainty about erroneous attributions can lead to delays in seeking care for preterm labor symptoms. Early certainty may turn to uncertainty about the attribution as the symptom pattern evolves.

\section{Limitations}

The results of this study should be interpreted with the recognition that the data were collected in a single hospital where the admission diagnosis of preterm labor was made by members of a medical staff with diverse opinions about the definition of preterm labor and the management of preterm symptoms. Some of the women in this study met the diagnostic criteria for preterm labor. Others were hospitalized for symptoms that did not strictly meet diagnostic criteria for frequency of contractions and cervical change. These women were, however, experiencing preterm symptoms that caused them to seek health care. Creasy and Resnik (1999) noted that cervical change of $1 \mathrm{~cm}$, dilation of $2 \mathrm{~cm}$, or a positive fetal fibronectin level should be documented before a diagnosis of preterm labor is made. Because records of the women's office visits were not available for this study, it was not possible to find documentation of cervical change from a previous examination. Contraction frequency of four contractions in 20 minutes or eight contractions in 1 hour are additional criteria for the diagnosis of preterm labor (Gonik \& Creasy, 1986). In the documentation reviewed for this study, contractions often were recorded as irregular rather than as a detailed contraction count.

Data for this study were based on recollections of women's experiences over the previous

\section{Weiss, Saks, \& Harris}


days and weeks. The accuracy of these recollections cannot be assessed, although the women were able to tell their stories vividly, in detail, and with clarity in terms of times and events. The results of this study, therefore, should not be generalized beyond the study population. In addition, it should be recognized that the results relate to women's experiences with preterm symptoms but not necessarily preterm labor.

\section{Implications for Perinatal Care}

This study has significant implications for perinatal care. First, the language used by expectant women in their descriptions of preterm labor symptoms should be incorporated in the educational materials available to all pregnant women to assist them in the early recognition of preterm labor. In particular, every expectant woman needs education about the cues to use in recognition of preterm labor as differentiated from term labor. The women in this study clearly identified the symptoms as qualitatively different than those experienced with term labor.

Furthermore, patient education should be directed not only toward providing materials to assist in recognizing and attributing symptom experiences to preterm labor but also to increasing awareness among pregnant women of the possibility of preterm labor. Health educators must balance the need to demedicalize the normal process of pregnancy and birth with the need to maintain sensitivity among expectant women of the possibilities of health symptoms requiring their attention and action. Health education for pregnant women should focus on health promotion and risk reduction as well as a proactive vigilance in self-monitoring for the early detection of problems. Expectant women should be given decision guides to assist them with self-management, timely contacts with the provider, and access points for seeking health care.

Early intervention for preterm labor requires recognition of the subtle cues by both the expectant woman and her health care provider. Women need access to providers who are sensitive to these cues and provide appropriate information and follow-up for women with prodromal symptoms who seek care. Sensitivity to the subtle differences between normal pregnancy sensations and the prodromal symptoms of preterm labor is a key factor in assuring timely access to care. The dilemma with preterm symptoms is that many symptom experiences do not result in the onset of preterm labor. Patients, however, should be encouraged to see their health care providers for clarification and verification of their symptom patterns.

The provider's office staff must be knowledgeable and sensitive to preterm labor symptoms. Women frequently interact with nonlicensed office staff who may not have the knowledge or the skills to make appropriate decisions about patient needs. Mechanisms need to 
be put in place to triage calls regarding preterm symptoms, provide consistent follow-up, and record the progress of symptom patterns so that risk reduction strategies can be implemented, onset can be recognized at the earliest possible point, and intervention can begin if preterm labor occurs.

Patient contacts for care related to preterm symptoms must be tracked over time rather than treating each encounter as an independent episode of care. These tracking mechanisms can build on the expectant woman's natural sense of vigilance about the pregnancy. Just as expectant women synthesize the multiple symptom cues to preterm labor onset, so must the health care provider. Computerized information systems that track the patient longitudinally across multiple encounters and settings can assist the provider in identifying expectant women who demonstrate a pattern of symptoms suggestive of a risk of preterm labor onset.

This and other studies (Cooper et al., 1990; lams, Stilson, et al., 1990; Katz et al., 1986) have documented symptoms of preterm labor that may occur a few hours to a few weeks before the onset of active preterm labor, suggesting that a prodromal phase may herald progression to an active phase that meets diagnostic criteria. Research to date has focused on the documentation of symptoms in this period, but further research is needed to determine strategies for identification and appropriate management of prodromal symptom patterns. The use of biochemical markers such as fetal fibronectin and salivary estriol appears promising in identifying women at risk for preterm labor (Leitich et al., 1999; McGregor et al., 1995). These technologies will support rather than replace the critical role of early recognition of symptom patterns by the expectant woman and her health care provider. This study also points to the need for further systematic investigation of factors associated with delays in recognizing and seeking care for preterm labor symptoms. The critical interface between the health care provider and women with preterm symptoms offers a unique opportunity to investigate the facilitators and barriers to early detection and treatment for preterm labor. Understanding patterns, sources, and consequences of delays will position health care scientists to define and test effective strategies for improving recognition, reducing delay in care seeking, and enhancing pregnancy outcomes.

\section{Acknowledgment}

- This study was supported in part by a research grant from the Association of Women's Health, Obstetric and Neonatal Nurses.

\section{Notes}

17 Weiss, Saks, \& Harris 
- Marianne E. Weiss is an associate professor of nursing, Marquette University, Milwaukee, WI.

- Nancy P. Saks is chair, Department of Nursing, National University, La Jolla, CA.

- Susan Harris is provost and vice president, Academic Affairs, National University, La Jolla, CA.

- Address for correspondence: Marianne E. Weiss, RN, DNSc, Marquette University College of Nursing, Milwaukee, WI 53201-1881. E-mail: marianne.weiss@marquette.edu.

\section{References}

Collaborative Group on Preterm Birth Prevention. (1993). Multicenter randomized, controlled trial of preterm birth prevention program. American Journal of Obstetrics and Gynecology, 169, 352-366.

Cooper, R. L., Goldenberg, R. L., Davis, R. O., Cutter, G. R., DuBard, M. B., Corliss, D. K., \& Andrews, J. B. (1990). Warning symptoms, uterine contractions, and cervical examination findings in women at risk for preterm delivery. American Journal of Obstetrics and Gynecology, 162, 748-754.

Coster-Schulz, M. A., \& Mackey, M. C. (1998). The preterm labor experience: A balancing act. Clinical Nursing Research, 7(4), 335-362.

Creasy, R. K., \& Resnik. R (1999). Maternal-fetal medicine. Philadelphia: W. B. Saunders.

Dyson, D. C., Danbe, K. H., Bamber, J. A., Crites, Y. M., Field, D. R., Maier, J. A., et al. (1998). Monitoring women at risk for preterm labor. New England Journal of Medicine, 338(1), 15-19.

Freston, M., Young, S., Calhoun, S., Frederickson, T., Salinger, L., Malchodi, C., \& Egan, J.F.X. (1997). Responses of pregnant women to potential preterm labor symptoms. Journal of Obstetric, Gynecologic, and Neonatal Nursing, 26, 35-42.

Furnham, A. F (1988). Lay theories: Everyday understanding of problems in the social sciences. New York: Pergamon.

Gonik, B., \& Creasy, R. K. (1986). Preterm labor: Its diagnosis and management. American Journal of Obstetrics and Gynecology, 154, 3-8.

Holbrook, R. H., Falcon, J., Herron, M., Lirette, M., Laros, R. K., \& Creasy, R. K. (1987). Evaluation of the weekly cervical examination in a preterm birth prevention program. American Journal of Perinatology, 4(3), 240-244.

lams, J. D., Johnson, F. F., \& Hamer, C. (1990). Uterine activity and symptoms as predictors of 18 Weiss, Saks, \& Harris 
preterm labor. Obstetrics and Gynecology, 76(Suppl.), 42-46.

lams, J. D., Johnson, F. F., \& O'Shaughnessy, R.W. (1988). A prospective random trial of home uterine activity monitoring in pregnancies with increased risk of preterm labor. American Journal of Obstetrics and Gynecology, 159, 595-603.

lams, J. D., Johnson, F. F., \& Parker, M. (1994). A prospective evaluation of signs and symptoms of preterm labor. Obstetrics and Gynecology, 84, 227-230.

lams, J. D., Stilson, R., Johnson, F. F., Williams, R. A., \& Rice, R. (1990). Symptoms that precede preterm labor and preterm premature rupture of the membranes. American Journal of Obstetrics and Gynecology, 162, 486-490.

Jaspers, A. (1983). Attribution theory and research. In J. Jaspers, F. Fincham, \& M. Hewstone (Eds.), Attribution theory and research. London: Academic Press.

Katz, M., Goodyear, K., \& Creasy, R. (1990). Early signs and symptoms of preterm labor. American Journal of Obstetrics and Gynecology, 162, 1150-1153.

Katz, M., Newman, R. B., \& Gill, P. J. (1986). Assessment of uterine activity in ambulatory patients at high risk for preterm labor and delivery. American Journal of Obstetrics and Gynecology, 154, 44-47.

Kelley, H. (1971). Attribution in social interaction. Morristown, NJ: General Learning.

Kragt, H., \& Keirse, M.J.N.C. (1990). How accurate is the woman's diagnosis of threatened preterm delivery? British Journal of Obstetrics and Gynaecology, 97, 317-323.

Leitich, H., Egarter, C., Kaider, A., Hohlagschwandtner, M., Berghammer, P., \& Husslein, P. (1999). Cervicovaginal fetal fibronectin as a marker for preterm delivery: A meta-analysis. American Journal of Obstetrics and Gynecology, 180(5), 1169-1176.

Mackey, M. C., \& Coster-Schulz, M. A. (1992). Women's views of the preterm labor experience. Clinical Nursing Research, 1, 366-384.

Maloni, J. A. (2000). Preventing preterm birth: Evidence-based interventions shift toward prevention. AWHONN Lifelines, 4(4), 26-33.

McGregor, J. A., Jackson, G. M., Lachelin, G. C., Goodwin, T. M., Artal, R., Hastings, C., \& Dullien, V. (1995). Salivary estriol as risk assessment for preterm labor: A prospective trial. American Journal of Obstetrics and Gynecology, 173(4), 1337-1342.

Mishel, M. R. (1988). Uncertainty in illness. Image: Journal of Nursing Scholarship, 20, 225-232. Nagey, D. A., Bailey-Jones, C., \& Herman, A. A. (1993). Randomized comparison of home uterine activity monitoring and routine care in patients discharged after treatment for preterm labor. Obstetrics \& Gynecology, 82(3), 319-323.

19 Weiss, Saks, \& Harris 
Newman, R. B., Gill, P., Wittreich, P., \& Katz, M. (1986). Maternal perception of prelabor uterine activity. Obstetrics and Gynecology, 68, 765-769.

Patterson, E. T., Douglas, A. B., Patterson, P. M., \& Bradle, J. B. (1992). Symptoms of preterm labor and self-diagnostic confusion. Nursing Research, 41, 367-372.

Pendleton, D. (1983). Doctor-patient communication: A review. In D. Pendleton \& J. Hasler (Eds.), Doctor-patient communication. London: Academic Press.

Shaver, K. G. (1983). An introduction to attribution processes. Hillsdale, NJ: Lawrence Erlbaum.

Thorne, S., Kirkham, S. R., \& MacDonald-Emes, J. (1997). Interpretive description: A non-categorical qualitative alternative for developing nursing knowledge. Research in Nursing and Health, 20, 169-177.

U.S. Preventive Services Task Force. (1993). Home uterine activity monitoring for preterm labor. Journal of the American Medical Association, 270, 369-376.

Ventura, S. J., Martin, J. A., Curtin, S. C., Mathews, T. J., \& Park, M. M. (2000). Birth: Final data for 1998. National Vital Statistics Report, 48(3), 1-100.

Watts, F. N. (1982). Attributional aspects of medicine. In C. Antaki \& C. Brewin (Eds.), Attributions and psychological change. London: Academic Press.

Weiner, B. (1979). A theory of motivation for some classroom experiences. Journal of Educational Psychology, 71, 3-25.

20 Weiss, Saks, \& Harris 


\section{Appendix}

Table 1

A Comparison of Uncertainty in IIIness Theory and Themes from Resolving the Uncertainty of Preterm Symptoms

\begin{tabular}{|l|l|}
\hline Uncertainty in IIIness Theory $^{\mathbf{a}}$ & $\begin{array}{l}\text { Resolving the Uncertainty of Preterm } \\
\text { Symptoms }\end{array}$ \\
\hline $\begin{array}{l}\text { Stimuli frame } \\
\text { Symptom patter } \\
\text { Event congruity }\end{array}$ & $\begin{array}{l}\text { Recognizing and naming symptoms } \\
\text { Attributing symptoms }\end{array}$ \\
\hline Uncertainty & Certainty/uncertainty of symptom attribution \\
\hline $\begin{array}{l}\text { Inference } \\
\text { Danger or opportunity }\end{array}$ & Perceived threat \\
\hline Coping strategies & $\begin{array}{l}\text { Interpreting/verifying symptom meaning } \\
\text { Managing symptoms } \\
\text { Engaging health care assistance }\end{array}$ \\
\hline Adaptation & $\begin{array}{l}\text { Admission for treatment } \\
\text { Delay in delivery or preterm birth }\end{array}$ \\
\hline
\end{tabular}

${ }^{a}$ Mishel (1988).

21 Weiss, Saks, \& Harris 